\title{
Risk factors of chronic left ventricular dysfunction after cardiac valve surgery
}

\author{
Xingli Fan", Yangfeng Tang", Guanxin Zhang", Jiajun Zhang, Qing Xue, Boyao Zhang, Zhiyun Xu, Lin Han \\ Department of Cardiovascular Surgery, Changhai Hospital, Naval Medical University, Shanghai, China \\ Contributions: (I) Conception and design: L Han; (II) Administrative support: Z Xu; (III) Provision of study materials or patients: L Han, Z Xu; (IV) \\ Collection and assembly of data: X Fan, Y Tang, G Zhang; (V) Data analysis and interpretation: X Fan, B Zhang, Q Xue, J Zhang; (VI) Manuscript \\ writing: All authors; (VII) Final approval of manuscript: All authors. \\ \#These authors contributed equally to this work. \\ Correspondence to: Lin Han. Department of Cardiovascular Surgery, Changhai Hospital, Naval Medical University, Shanghai, China. \\ Email: sh_hanlin@163.com.
}

Background: To analyze the risk factors of chronic left ventricular dysfunction (LVD) after cardiac valve surgery.

Methods: A retrospective analysis of 860 patients who underwent heart valve surgery in our center from January 2017 to December 2018, including 650 males and 210 females, aged 58 \pm 5.8 years. Inclusion criteria: (I) the patient was clinically diagnosed with heart valve disease and met the surgical indications for mitral valve replacement (MVR), mitral valve repair (MVP), aortic valve replacement (AVR) and double valve replacement (DVR); (II) if atrial fibrillation, coronary artery disease, and tricuspid regurgitation are combined before surgery, radiofrequency ablation, coronary bypass and tricuspid angioplasty were performed contemporarily. Exclusion criteria: (I) preoperative LVEF <50\%; (II) aortic dissection underwent Bentall and right heart valve replacement procedures; (III) cardiopulmonary resuscitation and death during perioperative period and 6 months after operation; (IV) postoperative CRRT, IABP, or ECMO assistance; (V) postoperative cardiac dysfunction due to valvular dysfunction, perivalvular leak, or infective endocarditis. Patients were divided into LVD group (LVEF < 40\%) and control group (LVEF $\geq 40 \%$ ) based on cardiac LVEF at 6 months after surgery. Logistic regression was used to analyze the risk factors of postoperative LVD.

Results: There were 126 cases in LVD group and 734 cases in control group. There were significant differences in preoperative coronary artery disease, atrial fibrillation, pulmonary hypertension, NYHA classification, left ventricular end diastolic diameter (LVEDD), and left ventricular end systolic diameter (LVESD) between the two groups $(\mathrm{P}<0.05)$. The differences in the changes of LVEDD and LVESD before and after operation between the two groups were statistically significant $(\mathrm{P}<0.05)$. Logistic regression analysis showed that preoperative LVEDD $>55 \mathrm{~mm}$, preoperative LVESD $>40 \mathrm{~mm}$, preoperative combined atrial fibrillation, preoperative combined pulmonary hypertension, preoperative NYHA III-IV, and preoperative combined coronary artery disease were the risks of postoperative chronic LVD.

Conclusions: The left ventricular diameter, preoperative coronary artery disease, NYHA III-IV, preoperative atrial fibrillation, and preoperative pulmonary hypertension are risk factors for chronic LVD after heart valve surgery.

Keywords: Heart valve surgery; left ventricular dysfunction (LVD); atrial fibrillation; pulmonary hypertension

Submitted Feb 25, 2020. Accepted for publication Jul 22, 2020.

doi: $10.21037 /$ jtd-20-1010

View this article at: http://dx.doi.org/10.21037/jtd-20-1010 


\section{Introduction}

Surgery is an important method for the treatment of heart valve disease, but some patients have chronic left ventricular dysfunction (LVD) after operation, which seriously affects the quality of life of patients and reduces long-term survival rate. Previous studies have shown that preoperative and intraoperative factors can lead to the occurrence of chronic LVD after heart valve surgery, but most of them are risk factor analysis of chronic LVD after homogeneous valve surgery $(1,2)$. This study aimed to analysis the risk factors of postoperative chronic LVD after heart valve surgery. We present the following article in accordance with the STROBE reporting checklist (available at http://dx. doi. org/10. 21037/jtd-20-1010).

\section{Methods}

A retrospective analysis of 860 patients who underwent heart valve surgery in our center from January 2017 to December 2018, including 650 males and 210 females, aged $58 \pm 5.8$ years. Inclusion criteria: (I) the patient was clinically diagnosed with heart valve disease and met the surgical indications for mitral valve replacement (MVR), mitral valve repair (MVP), and aortic valve replacement (AVR) and double valve replacement (DVR); (II) if atrial fibrillation, coronary artery disease, and tricuspid regurgitation are combined before surgery, the same period of time can be achieved during the operation. Radiofrequency ablation, coronary bypass and tricuspid angioplasty were performed. Exclusion criteria: (I) preoperative LVEF $<50 \%$; (II) aortic dissection with Bentall and right heart valve replacement; (III) cardiopulmonary resuscitation and death during perioperative period and 6 months after operation; (IV) operation post-CRRT, IABP, or ECMO mechanical assistance; (V) postoperative cardiac dysfunction due to valvular dysfunction, perivalvular leak, or infective endocarditis. All patients were reviewed for color Doppler ultrasound at 30 days and 6 months after operation. Patients were divided into LVD group (LVEF <40\%) and control group (LVEF $\geq 40 \%$ ) according to LVEF at 6 months after operation.

Warfarin anticoagulation was taken after all simple valve surgery, including lifelong anticoagulation after mechanical valve, 6 months after biological valve and mitral valve angioplasty, and warfarin combined with aspirin anticoagulation after coronary bypass surgery. Patients undergoing radiofrequency ablation of atrial fibrillation during the operation were given amiodarone for 3 months to control the heart rate. Cardiac ultrasound was routinely followed up at 30 days and 6 months after the operation.

All data were statistically analyzed using SAS v9.1 software. Continuous variables were expressed as mean + standard deviation, categorical variables were expressed as percentages, differences between groups were analyzed by $t$ test or Wilcoxon rank sum test, and differences between groups of categorical variables were analyzed by $\mathrm{x}^{2}$ test. Logistic regression was used to analyze the risk factors of postoperative LVD. The difference was statistically significant at $\mathrm{P}<0.05$.

The retrospective study was approved by the Ethics Committee of Changhai Hospital affiliated to Naval Medical University (No. 20200219) and informed consent was taken from all the patients. The study was conformed to the provisions of the Declaration of Helsinki (as revised in 2013).

\section{Results}

There were 126 cases in the LVD group and 734 cases in the control group. There was no significant difference in age, sex, smoking history, weight, preoperative hypertension, diabetes, and renal insufficiency between the two groups $(\mathrm{P}>0.05)$. There was a statistically significant difference in the combination of coronary artery disease, atrial fibrillation, pulmonary hypertension, and NYHA classification $(\mathrm{P}<0.05)$ (Table 1).

There was no statistically significant difference in the thickness of the ventricular septum, the thickness of the posterior wall of the left ventricle, the number of surgical operations at each valve position, the concurrent coronary bypass, tricuspid valve angioplasty, and radiofrequency ablation of atrial fibrillation, and the block time and extracorporeal circulation time were not significantly different between the two groups $(\mathrm{P}>0.05)$; there was a statistically significant difference in the LVEDD and LVESD between the two groups $(\mathrm{P}<0.05)$; there was a statistically significant difference in the changes in the LVEDD and LVESD before and after the two groups $(\mathrm{P}<0.05)$ (Table 2).

Logistic regression analysis showed that preoperative LVEDD $>55 \mathrm{~mm}(\mathrm{OR}=3.342$, 95\% CI: $1.789-7.334$, $\mathrm{P}=0.018)$, preoperative LVESD $>40 \mathrm{~mm}(\mathrm{OR}=3.181,95 \%$ CI: $1.675-7.245, \mathrm{P}=0.020)$, Preoperative atrial fibrillation $(\mathrm{OR}=5.123,95 \%$ CI: 0.245-10.332, $\mathrm{P}=0.009)$, preoperative 
Table 1 Preoperative baseline demographic data

\begin{tabular}{lccc}
\hline Variables & LVD group $(\mathrm{LVEF}<40 \%, \mathrm{n}=126)$ & Control group $(\mathrm{LVEF} \geq 40 \%, \mathrm{n}=734)$ & $\mathrm{P}$ value \\
\hline Age (years) & $57 \pm 14.7$ & $59 \pm 10.7$ & 0.513 \\
Male (\%) & $98(77.8)$ & $551(75.1)$ & 0.704 \\
Smoke (\%) & $59(46.8)$ & $347(47.3)$ & 0.672 \\
Weight (kg) & $65 \pm 12.5$ & $69 \pm 10.7$ & 0.543 \\
Hypertension & $44(34.9)$ & $293(39.9)$ & 0.235 \\
Diabetes & $37(29.4)$ & $190(25.9)$ & 0.356 \\
CAD & $26(20.6)$ & $88(12.0)$ & 0.006 \\
Renal dysfunction & $12(9.5)$ & $58(7.9)$ & 0.482 \\
AF & $50(39.7)$ & $211(28.7)$ & 0.007 \\
PHT & $44(34.9)$ & $183(24.9)$ & 0.010 \\
NYHA classifications & & & $<0.001$ \\
I-II & $63(50.0)$ & $491(66.9)$ & 0.012 \\
III & $34(27.0)$ & $132(18.0)$ & 0.001 \\
IV & $29(23.0)$ & $106(14.4)$ & \\
\hline
\end{tabular}

LVD, left ventricular dysfunction; CAD, coronary artery disease; AF, atrial fibrillation; PHT, pulmonary hypertension; NYHA, New York heart association.

pulmonary hypertension $(\mathrm{OR}=4.994,95 \% \mathrm{CI}: 0.564-$ 11.356, $\mathrm{P}=0.012$ ), preoperative NYHA III-IV Grade (OR $=2.762,95 \%$ CI: $1.022-4.234, \mathrm{P}=0.023)$ and preoperative coronary disease $(\mathrm{OR}=3.566,95 \% \mathrm{CI}: 1.973-8.013$, $\mathrm{P}=0.028$ ) are risk factors for postoperative LVD (Table 3).

\section{Discussion}

Complications of LVD after heart valve surgery seriously affect patients' quality of life and long-term survival rate. This study included all heart valve surgery cases analyzed in our center within two years, and the results showed that compared with the control group, the incidence of major cardiovascular events and rehospitalization rate of patients with LVD after heart valve surgery were higher. Risk factors for LVD after heart valve surgery include left ventricular size, atrial fibrillation, pulmonary hypertension, NYHA III$\mathrm{IV}$, and preoperative coronary artery disease.

Chan et al. Found that preoperative left ventricular dilatation and pulmonary hypertension were risk factors for cardiac insufficiency after mitral angioplasty, and thought that in symptomatic patients, once the left ventricular end-systolic diameter index was $>19 \mathrm{~mm} / \mathrm{m}^{2}$, right ventricular systolic pressure was $>45 \mathrm{mmHg}$ should be actively operated to reduce the incidence of postoperative LVD (3). Amano et al. Analyzed 58 patients with AVR, of which 21 (36\%) had preoperative LVEF $<50 \%$, and $7(12 \%)$ had secondary LVD after operation. The left ventricular diameter increased significantly before operation in the LVD group, suggesting that Increased left ventricular diameter is associated with postoperative LVD (4). The left ventricular diameter of all patients in this study was significantly lower than that before surgery, suggesting that surgery can delay or prevent myocardial remodeling caused by valve disease, reduce heart load, and improve heart function. We found that preoperative left ventricular diameter increase is a risk factor for LVD after valve operation, which may be related to pre-existing cardiac dysfunction. It is more common in mitral valve insufficiency, some in aortic valve insufficiency, and mitral valve or Patients with aortic stenosis are rare. Patients with mitral and aortic valve insufficiency are accompanied by increased cardiac volume load and eccentric hypertrophy, and the left ventricular end-diastolic diameter increases, but at this time, the diameter and systolic function are normal, and LVEF remains in the normal range. As the volume load continues Increased, patients with increased left ventricular end-systolic diameter and myocardial 
Table 2 Echocardiography and intraoperative data

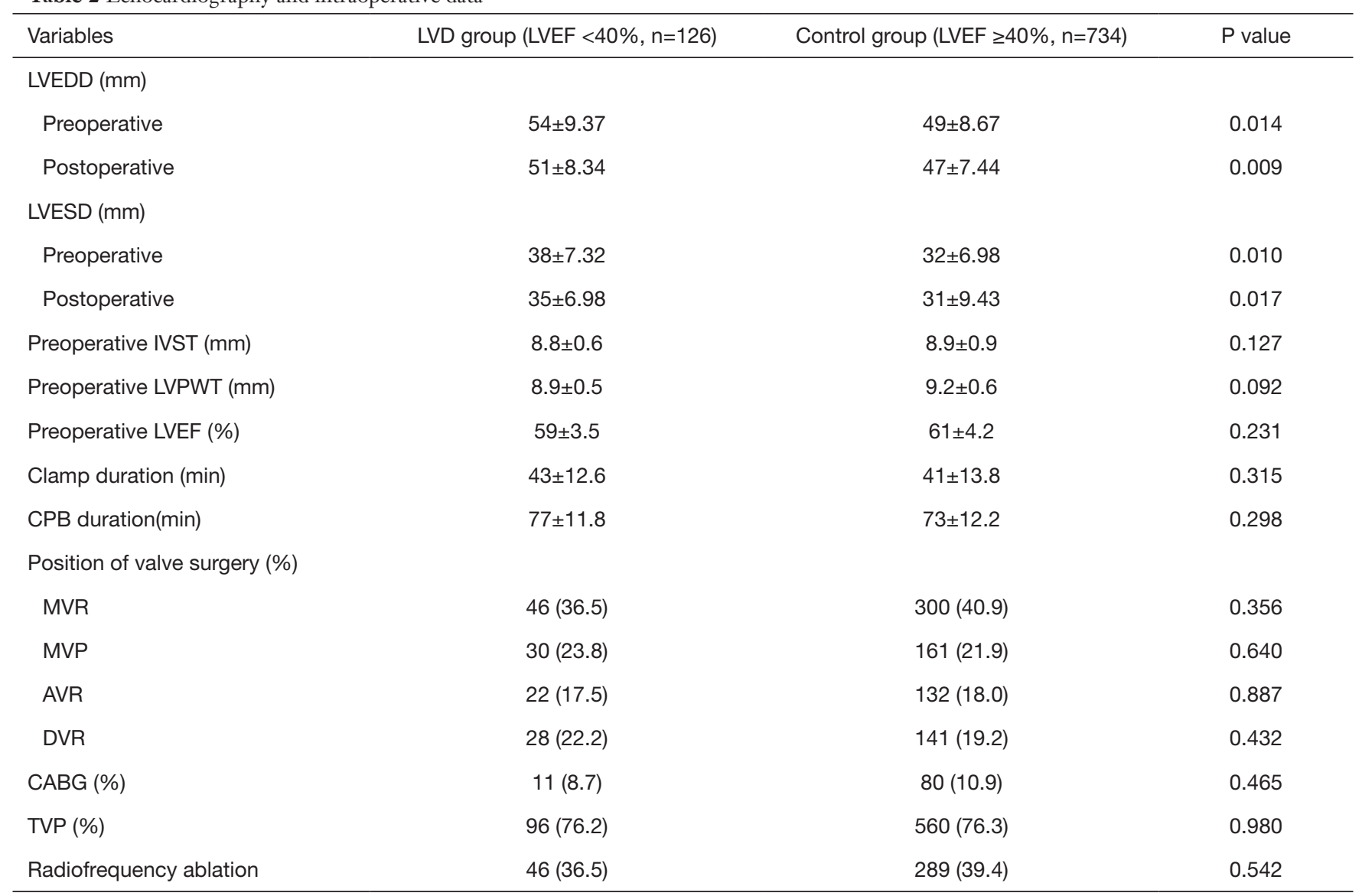

LVD, left ventricular dysfunction; LVEDD, left ventricular end diastolic diameter; LVESD, left ventricular end systolic diameter; IVST, interventricular septum thickness; LVPWT, left ventricular posterior wall thickness; LVEF, left ventricular ejection fraction; CPB, cardiopulmonary bypass; MVR, mitral valve replacement; MVP, mitral valvuloplasty; AVR, aortic valve replacement; DVR, double valve replacement; CABG, coronary artery bypass graft; TVP, tricuspid valvuloplasty.

Table 3 Predicts of postoperative chronic left ventricular dysfunction

\begin{tabular}{lccc}
\hline Variables & OR value & $95 \%$ Cl & P value \\
\hline Preoperative LVEDD $>55 \mathrm{~mm}$ & 3.242 & $1.789-7.334$ & 0.018 \\
Preoperative LVESD $>40 \mathrm{~mm}$ & 3.181 & $1.675-7.245$ & 0.020 \\
Preoperative AF & 5.123 & $0.245-10.345$ & 0.009 \\
Preoperative PHT & 4.994 & $0.564-11.356$ & 0.012 \\
Preoperative NYHA III-IV & 2.762 & $1.022-4.234$ & 0.023 \\
CPB duration & 1.185 & $0.1234-3.432$ & 0.084 \\
Preoperative CAD & 3.566 & $1.973-8.013$ & 0.028 \\
\hline
\end{tabular}

LVEDD, left ventricular end diastolic diameter; LVESD, left ventricular end systolic diameter; AF, atrial fibrillation; PHT, pulmonary hypertension; NYHA, New York heart association; CPB, cardiopulmonary bypass; CAD, coronary artery disease. 
dysfunction, LVEF began to decline. If valvular surgery can reduce the heart volume load at this time, the existing myocardial dysfunction and lower postoperative preload can lead to a decrease in postoperative LVEF. Therefore, we believe that reasonable choice of surgical timing and early intervention can reduce postoperative incidence of LVD.

Studies have shown that atrial fibrillation is an independent predictor of long-term survival and LVD after mitral valve angioplasty, and that patients with mitral regurgitation should be operated immediately once atrial fibrillation is complicated (5). A study of 632 patients with mitral angioplasty considered atrial fibrillation to be a sign of the severity of valvular regurgitation. Preoperative combined atrial fibrillation and pulmonary hypertension doubled the incidence of early postoperative LVD (6). The above study population were all patients who underwent mitral valve angioplasty. This analysis of all valve operations still suggests that atrial fibrillation is a risk factor for LVD after valvular surgery. Regarding its specific mechanism, we believe that atrial fibrillation causes atrial assisted ventricular filling to disappear the irregular rhythm of the ventricle causes ventricular systolic dysfunction. Therefore, surgical intervention for valvular disease with early atrial fibrillation or even before the occurrence of atrial fibrillation can reduce the rate of complications and improve long-term survival.

Pulmonary hypertension often indicates that the prevalence of valvular disease is severe (7), and the onset time is long. Although the left ventricular function of the patient is relieved after valve operation, continuous pulmonary hypertension damages the right ventricular function, and the reduction of left ventricular preload leads to a decrease in LVEF. Preoperative complications of coronary artery disease and NYHA grades III to IV indicate that there may be cardiac dysfunction before the ejection fraction retention, which is more susceptible to myocardial ischemia-reperfusion injury, and therefore LVD is more likely to occur after surgery.

\section{Conclusions}

In summary, large left ventricular diameter, coronary artery disease, NYHA III-IV, atrial fibrillation, and pulmonary hypertension are risk factors for chronic LVD after heart valve surgery. Valvular disease leads to myocardial remodeling. Actively performing reverse myocardial remodeling before surgery and choosing the right time for surgery to prevent myocardial remodeling can reduce the incidence of postoperative LVD. If patients with atrial fibrillation, pulmonary hypertension, and coronary disease, and reasonable indications of concurrent radiofrequency ablation and coronary bypass surgery during surgery, can reduce the incidence of postoperative arrhythmia, improve myocardial blood supply, and lower the pulmonary artery when necessary Stress treatment may be an important measure to reduce the incidence of postoperative LVD.

\section{Acknowledgments}

Funding: None.

\section{Footnote}

Reporting Checklist: The authors have completed the STROBE reporting checklist. Available at http://dx.doi. org/10.21037/jtd-20-1010

Data Sharing Statement: Available at http://dx.doi. org/10.21037/jtd-20-1010

Conflicts of Interest: All authors have completed the ICMJE uniform disclosure form (available at http://dx.doi. org/10.21037/jtd-20-1010). The authors have no conflicts of interest to declare.

Ethical Statement: The authors are accountable for all aspects of the work in ensuring that questions related to the accuracy or integrity of any part of the work are appropriately investigated and resolved. The retrospective study was approved by the Ethics Committee of Changhai Hospital affiliated to Naval Medical University (No. 20200219) and informed consent was taken from all the patients. The study was conformed to the provisions of the Declaration of Helsinki (as revised in 2013).

Open Access Statement: This is an Open Access article distributed in accordance with the Creative Commons Attribution-NonCommercial-NoDerivs 4.0 International License (CC BY-NC-ND 4.0), which permits the noncommercial replication and distribution of the article with the strict proviso that no changes or edits are made and the original work is properly cited (including links to both the formal publication through the relevant DOI and the license). See: https://creativecommons.org/licenses/by-nc-nd/4.0/. 


\section{References}

1. Gentles TL, French JK, Zeng I, et al. Normalized endsystolic volume and pre-load reserve predict ventricular dysfunction following surgery for aortic regurgitation independent of body size. JACC Cardiovasc Imaging 2012;5:626-33.

2. Witkowski TG, Thomas JD, Debonnaire PJMR, et al. Global longitudinal strain predicts left ventricular dysfunction after mitral valve repair. Eur Heart J Cardiovasc Imaging 2013;14: 69-76.

3. Chan V, Ruel M, Elmistekawy E, et al. Determinants of left ventricular dysfunction after repair of chronic asymptomatic mitral regurgitation. Ann Thorac Surg 2015;99:38-42.

4. Amano M, Izumi C, Imamura S, et al. Late recurrence of

Cite this article as: Fan X, Tang Y, Zhang G, Zhang J, Xue Q, Zhang B, Xu Z, Han L. Risk factors of chronic left ventricular dysfunction after cardiac valve surgery. J Thorac Dis 2020;12(9):4854-4859. doi: 10.21037/jtd-20-1010 left ventricular dysfunction after aortic valve replacement for severe chronic aortic regurgitation. Int J Cardiol 2016;224:240-4.

5. Szymanski C, Magne J, Fournier A, et al. Usefulness of preoperative atrial fibrillation to predict outcome and left ventricular dysfunction after valve repair for mitral valve prolapse. Am J Cardiol 2015;115:1448-53.

6. Ruel M, Rubens FD, Masters RG, et al. Late incidence and predictors of persistent or recurrent heart failure in patients with mitral prosthetic valves. J Thorac Cardiovasc Surg 2004;128:278-83.

7. Varghese R, Itagaki S, Anyanwu AC, et al. Predicting early left ventricular dysfunction after mitral valve reconstruction: the effect of atrial fibrillation and pulmonary hypertension. J Thorac Cardiovasc Surg 2014;148:422-7. 